The Egyptian Journal of Hospital Medicine (January 2019) Vol. 74 (2), Page 388-395

\title{
Assessment of Procalcitonin Level in Chronic Hemodialysis Patients with Obesity
}

\author{
Mustafa Abd-Elfattah El-Ballat (1), El-Sayed Mohammed Rashid ${ }^{(1)}$, Abd-EIraouf Abd-Elraouf Abo \\ Nar ${ }^{(2)}$, Salah Fathi Ali Radwan ${ }^{(3)}$.
}

(1) Internal Medicine and Nephrology Department, (2) Clinical Pathology Department, Faculty of Medicine, Al-Azhar University, Cairo, (3) Nephrology Department, El-Sahel Teaching Hospital, Cairo, Egypt

Corresponding author: Salah Fathi Ali Radwan, email: dr.sradwan@yahoo.com

\begin{abstract}
Background: Circulating procalcitonin (PCT) is an inflammatory marker produced by several cell types including adipose tissue following cytokine stimulation. In contrast to its role in the general population, obesity has been associated with improved survival in chronic renal failure patients undergoing haemodialysis. The aim of our study was to evaluate the link between plasma PCT concentration and metabolic parameters of obesity in chronic hemodialysis patients.

Patients and methods: We determined PCT in 80 subjects; they were divided into: 30 obese, body mass index(BMI) $>28 \mathrm{Kg} / \mathrm{m}^{2}$, chronic renal failure patients' undergoing haemodialysis, 30 non obese, body mass index(BMI) $<28 \mathrm{Kg} / \mathrm{m}^{2}$, chronic renal failure patients' undergoing haemodialysis, 20 obese, body mass index(BMI) $>28 \mathrm{Kg} / \mathrm{m}^{2}$, normal healthy subjects as control. Complete clinical examination and laboratory investigations were done.

Results: Elevated plasma procalcitonin (PCT) levels, within the normal range, of the 80 subjects included was not dependent on anthropometric measures including body weight, height, body mass index and waist circumference. It was found to be correlated to age $(0.020, \mathrm{P}<0.05)$ and urea $(0.024, \mathrm{P}<0.05)$ in obese haemodialysis patients'.

Conclusion: Our findings based on community-based data showed that higher plasma procalcitonin levels in the normal range are associated with increased measures of obesity in chronic renal failure patients' undergoing haemodialysis. Because associations were not dependent on BMI, plasma procalcitonin may serve as a new marker for adipocyte dysfunction, chronic low-grade inflammation, or both.
\end{abstract}

Keywords: Procalcitonin, hemodialysis, obesity.

\section{Introduction}

Procalcitonin (PCT) was first described in 1993 as a diagnostic marker of infection. It is a polypeptide of 116 amino acids and has a molecular weight of $13 \mathrm{kDa}$. It is the precursor for the calcium homeostasis hormone, calcitonin. Procalcitonin circulates at very low concentrations in normal serum and is thought to be produced in physiological conditions by the neuroendocrine cells in the thyroid gland and $\operatorname{lungs}{ }^{(\mathbf{1})}$.

Procalcitonin (PCT) high levels are strongly associated with systemic bacterial infections ${ }^{(\mathbf{1 , 2})}$. and with the severity of illness ${ }^{(3)}$. It is produced in response to bacterial endotoxin and inflammatory host cytokines and may help in discriminating bacterial from viral infections ${ }^{(4)}$ and true bacteremia from contaminated blood cultures $^{(5)}$. It is known that Gram-positive or Gram-negative bacteria or fungi activate different Toll-like receptor (TLR) signaling pathways, resulting in production of different proinflammatory cytokines that ultimately stimulate PCT release ${ }^{(6)}$.

The reference value of procalcitonin in adults and children older than 72 hours is $0.15 \mathrm{ng} / \mathrm{mL}$ or less. Reference values have not been established in infants younger than 72 hours. In healthy adults, the reference range of procalcitonin is below the level of detection ${ }^{(7)}$. The half-life of procalcitonin 
is 25-30 hours (not significantly altered in individuals with renal dysfunction) ${ }^{(8)}$.

A significant number of patients who undergo dialysis are overweight or obese ${ }^{(9)}$. The presence of excess weight, especially visceral obesity contributes to the increased risk of metabolic and cardiovascular complications in patients with chronic kidney disease (CKD). The relationship between weight and outcome is complex among dialysis patients. The extra weight is usually associated with improved survival in this patient group $^{(\mathbf{1 0})}$.

Recent studies show that adipose tissue is capable of expressing and secreting procalcitonin ${ }^{(11)}$. So we hypothesize that plasma procalcitonin may be associated with measures of obesity.

\section{Methods}

\section{Patients Population}

This is a cross sectional study that was conducted in El-Sahel Teaching Hospital and Sayed Galal University Hospital after taking the approval of the ethical committee of internal medicine department. Informed consent was taken from all patients included in the study. This study included 80 subjects; they were divided into three groups, Group 1 included 30 obese (BMI $>28 \mathrm{Kg}$ $\left./ \mathrm{m}^{2}\right)$ CRF patients' undergoing regular hemodialysis (HD) three times weekly; each dialysis session was performed in 4 hours. Blood access was through arterio-venous fistula (AVF). The duration of hemodialysis ranged between 0.8 and 13 years with a mean of $4.25 \pm 2.5$ years. Their age ranged between $39-75$ years with a mean of $56.47 \pm 10.04$ years. 16 of them were males and 14 were females. The dialyzers used were polyflux 1.6 (surface area $1.6 \mathrm{~m} 2$ ), polyflux 1.8(surface area $1.8 \mathrm{~m} 2$ ), Gambro 1.7 (surface area $1.7 \mathrm{~m} 2$ ) and polyflux 20 (surface area $2.0 \mathrm{~m} 2$ ) according to body weight for each patient. We used bicarbonate dialysis concentrate with a final concentration of diluted solution in mmol. /Liter as follows: Sodium 103mmol. / Liter, Magnesium $0.5 \mathrm{mmol}$. / Liter, Potassium 2mmol. / Liter, Chloride 109.5mmol. / Liter, Calcium 1.75mmol. / Liter, Bicarbonate 3mmol. / Liter. During dialysis the patients received intermittent heparinization with 10000 units as maximum dose.

Group 2 included 30 non obese $\left(\mathrm{BMI}<28 \mathrm{Kg} / \mathrm{m}^{2}\right)$ CRF patients' undergoing regular haemodialysis
(HD) three times weekly; each dialysis session was performed in 4 hours. Blood access was through arteriovenous fistula (AVF). The duration of haemodialysis ranged between 1 and 20 years with a mean of $4.50 \pm 3$ years. Their age ranged between $21-72$ years with a mean of $46.50 \pm 13.63$ years. 16 of them were males and 14 were females. The dialyzers used were platinum HF (surface area $1.4 \mathrm{~m} 2$ ),FX 60 (surface area $1.4 \mathrm{~m} 2$ ), polyflux 1.6(surface area $1.6 \mathrm{~m} 2$ ), polyflux 1.8 (surface area $1.8 \mathrm{~m} 2$ ) according to body weight for each patient.

Group 3 included 20 obese (BMI $>28 \mathrm{Kg} / \mathrm{m}^{2}$ ) normal healthy subjects as control. Six of them were males and 14 were females. Their age ranged between 29 - 63years with a mean of $43.13 \pm 10.78$ years.

\section{Laboratory Examinations}

Blood urea, serum creatinine, serum calcium, phosphorus, parathormone hormone (PTH), serum sodium, potassium, serum albumin, Creactive protein, complete blood count were measured at the beginning of HD. Anthropometric measures; Body weight, height, BMI and waist circumference were calculated.

Serum procalcitonin was determined by enzymelinked immunosorbent assay (ELISA). Serum sample were collected from the subjects (prior to dialysis as regard HD patients) then samples were allowed to clot for 10-20 minutes at room temperature, then samples were placed in centrifuge (at 2000-3000 RPM) for approximately 20 minutes. Supernatants were collected carefully. Samples were stored in $-20^{\circ}$ $\mathrm{C}$ temperature in refrigerator. If sediment had occurred during storage, centrifugation was repeated. The kit uses a double-antibody sandwich enzyme-linked immunosorbent assay (ELISA) to assay the level of Human Procalcitonin (PCT) in samples. Add Procalcitonin (PCT)to monoclonal antibody Enzyme well which is pre-coated with Human Procalcitonin (PCT) monoclonal antibody, incubation; then, add Procalcitonin (PCT)antibodies labeled with biotin, and combined with Streptavidin-HRP to form immune complex; then carry out incubation and washing again to remove the uncombined enzyme. Then add Chromogen Solution A, B, the 
color of the liquid changes into the blue, and at the effect of acid, the color finally becomes yellow. The Chroma of color and the concentration of the Human Substance Procalcitonin (PCT) of sample were positively correlated.

\section{Statistical Analysis}

The data were entered, coded and processed on computer using Statistical Packaged for Social Science (IBM SPSS). The level $\mathrm{p} \leq 0.05$ was considered the cut off value for significance. Description of quantitative variables as mean and standard deviation (SD).Description of qualitative variables as number and percentage .Chi-Square test $\chi^{2}$ was used to compare qualitative variables between groups. Independent samples t-test was used to assess the statistical significance of the difference between two population means in a study involving independent samples. One way ANOVA was used to assess the statistical significance of the difference in more than two population means in a study involving independent samples. Post Hoc Tests for situation in which there was a statistical significance of the difference in more than two populations means to tell us which groups differ from the rest. The correlation coefficient method was used to relate different parameters, person correlation for quantitative variables, and spearman correlation for ordinal variables. Probability $(\mathrm{P})$ value, $\mathrm{P}$ value was used as determinant as significance: If $\mathrm{P}>0.05=$ statistically Insignificant, If $\mathrm{P} \leq 0.05=$ statistically significant, If $\mathrm{P}<0.05=$ statistically highly significant. Graphic presentation of the results was also done.

\section{Results}

Our study showed that there was a statistical significant difference ( $\mathrm{P}$ value $<0.05$ ) between 3 group regarding the mean of diastolic blood pressure(DBP) and a highly statistical significant difference $(\mathrm{P}$ value <0.01) between 3 group regarding the mean of age,weight ,BMI, RBCs ,systolic blood pressure(SBP), urea ,Creatinine ,HB, $\mathrm{Ca}+$,Po4+ , albumin ,waist circumference(WC) ,PTH ,HCT and procalcitonin(PCT), however there was no a statistical significant difference $(\mathrm{P}$ value $>0.05$ ) between 3 group regarding the mean of sex ,height ,WBCs ,Na+ ,PLT ,CRP and potassium $(\mathrm{K}+)($ Table 1$)$.

\begin{tabular}{|c|c|c|c|c|c|c|c|c|c|}
\hline & \multirow{2}{*}{$\begin{array}{c}\text { Obese HD } \\
\text { No. }=\mathbf{3 0} \\
\end{array}$} & \multirow{2}{*}{\begin{tabular}{|c|}
$\begin{array}{c}\text { Non Obese } \\
\text { HD }\end{array}$ \\
No. $=\mathbf{3 0}$ \\
\end{tabular}} & \multirow{2}{*}{$\begin{array}{c}\begin{array}{c}\text { Normal } \\
\text { Obese }\end{array} \\
\text { No. }=20 \\
\end{array}$} & \multirow{2}{*}{$\begin{array}{c}\text { Test } \\
\text { value }\end{array}$} & \multirow{2}{*}{$\begin{array}{c}\text { P- } \\
\text { valu } \\
\text { e }\end{array}$} & \multicolumn{3}{|c|}{$\begin{array}{c}\text { Post Hoc analysis } \\
\text { by LSD }\end{array}$} \\
\hline & & & & & & & \begin{tabular}{|l|} 
P1 \\
\end{tabular} & $\mathbf{P 2}$ & $\mathbf{P 3}$ \\
\hline Age(years) & $\begin{array}{c}\text { Mean } \pm S \\
\text { D }\end{array}$ & $\begin{array}{l}56.47 \pm \\
10.04\end{array}$ & $\begin{array}{l}46.50 \pm \\
13.63\end{array}$ & $\begin{array}{c}43.13 \pm \\
10.78\end{array}$ & $9.290 \bullet$ & $\begin{array}{c}0,00 \\
1\end{array}$ & $\begin{array}{c}0.00 \\
1\end{array}$ & $\begin{array}{c}0,00 \\
1\end{array}$ & 0.320 \\
\hline $\mathrm{COH}$ & \begin{tabular}{|l|} 
Female \\
\end{tabular} & $14(46.7 \%)$ & $14(46.7 \%)$ & $14(70.0 \%)$ & $72 \%$ & 0.19 & 1.00 & 0.10 & 1 \\
\hline Sex & Male & $16(53.3 \%)$ & $16(53.3 \%)$ & $6(30.0 \%)$ & $2 / 3^{*}$ & 4 & 0 & 3 & .103 \\
\hline Weight $(\mathrm{kg})$ & $\begin{array}{c}\text { Mean } \pm S \\
D\end{array}$ & $\begin{array}{c}100.93 \pm \\
12.43\end{array}$ & $\begin{array}{c}65.82 \pm \\
16.87\end{array}$ & $87.43 \pm 5.41$ & $54.318^{\bullet}$ & $\begin{array}{c}0.00 \\
1\end{array}$ & $\begin{array}{c}0.00 \\
1\end{array}$ & $\begin{array}{c}0.00 \\
1\end{array}$ & 0.001 \\
\hline Height $(\mathrm{cm})$ & $\begin{array}{c}\text { Mean } \pm S \\
D\end{array}$ & $\begin{array}{c}170.77 \pm \\
4.87\end{array}$ & $\begin{array}{c}170.77 \pm \\
9.08\end{array}$ & $\begin{array}{c}167.45 \pm \\
3.83\end{array}$ & $1.891 \bullet$ & $\begin{array}{c}0.15 \\
8\end{array}$ & $\begin{array}{c}1.00 \\
0\end{array}$ & $\begin{array}{c}0.08 \\
6\end{array}$ & 0.086 \\
\hline BMI & \begin{tabular}{|c|} 
Mean $\pm S$ \\
$\mathrm{D}$ \\
\end{tabular} & 34.60 & $23.05 \pm 3.29$ & $31.11 \pm 1.91$ & $95.606^{\bullet}$ & $\begin{array}{c}0.00 \\
1\end{array}$ & $\begin{array}{c}0.00 \\
1 \\
\end{array}$ & $\begin{array}{c}0.00 \\
1 \\
\end{array}$ & 0.001 \\
\hline \begin{tabular}{|c|} 
WBCS \\
(thousand/cm \\
$\mathrm{m}$ ) \\
\end{tabular} & $\begin{array}{c}\text { Mean } \pm S \\
D\end{array}$ & $6.64 \pm 1.78$ & $7.08 \pm 1.95$ & $7.61 \pm 1.49$ & $1.786^{\bullet}$ & $\begin{array}{c}0.17 \\
5\end{array}$ & $\begin{array}{c}0.33 \\
8\end{array}$ & $\begin{array}{c}0.06 \\
3\end{array}$ & 0.310 \\
\hline \begin{tabular}{|c|} 
RBCS \\
(millions/cmm \\
)
\end{tabular} & $\begin{array}{c}\text { Mean } \pm S \\
D\end{array}$ & $3.62 \pm 0.55$ & $3.60 \pm 0.59$ & $4.19 \pm 0.47$ & $8.538 \bullet$ & $\begin{array}{c}0.00 \\
1\end{array}$ & $\begin{array}{c}0.88 \\
0\end{array}$ & $\begin{array}{c}0.00 \\
1\end{array}$ & 0.001 \\
\hline & & & $1 \mathrm{a}$ & & & & & & \\
\hline & & Obese HD & \begin{tabular}{|c|}
$\begin{array}{c}\text { Non Obese } \\
\text { HD }\end{array}$ \\
\end{tabular} & $\begin{array}{c}\text { Normal } \\
\text { Obese }\end{array}$ & $\begin{array}{c}\text { Test } \\
\text { value }\end{array}$ & & & $\begin{array}{l}\text { Ho } \\
\text { by }\end{array}$ & palysis \\
\hline
\end{tabular}


Mustafa El-Ballat et al.

\begin{tabular}{|c|c|c|c|c|c|c|c|c|c|}
\hline & & No. $=30$ & No. $=30$ & No. $=20$ & & $\begin{array}{c}\text { P- } \\
\text { valu } \\
\text { e }\end{array}$ & P1 & $\mathbf{P 2}$ & P3 \\
\hline $\mathrm{SBP}(\mathrm{mmHg})$ & $\begin{array}{c}\text { Mean } \pm S \\
\text { D }\end{array}$ & $\begin{array}{c}140.33 \pm \\
17.52\end{array}$ & $\begin{array}{c}127.33 \pm \\
19.64\end{array}$ & $\begin{array}{c}114.00 \pm \\
7.54\end{array}$ & $15.346^{\bullet}$ & $\begin{array}{c}0.00 \\
1\end{array}$ & \begin{tabular}{|c|}
0.00 \\
3
\end{tabular} & $\begin{array}{c}0.00 \\
1\end{array}$ & 0.007 \\
\hline $\mathrm{DBP}(\mathrm{mmHg})$ & $\begin{array}{c}\text { Mean } \pm S \\
\text { D }\end{array}$ & $84.33 \pm 7.28$ & $81.67 \pm 8.34$ & $78.00 \pm 4.10$ & $4.788 \bullet$ & $\begin{array}{c}0.01 \\
1\end{array}$ & \begin{tabular}{|c|}
0.14 \\
9
\end{tabular} & $\begin{array}{c}0.00 \\
3\end{array}$ & 0.077 \\
\hline $\operatorname{Urea}(\mathrm{mg} / \mathrm{dl})$ & $\begin{array}{c}\text { Mean } \pm S \\
\text { D }\end{array}$ & $\begin{array}{c}131.27 \pm \\
30.65\end{array}$ & $\begin{array}{c}152.00 \pm \\
31.14\end{array}$ & $\begin{array}{c}26.07 \pm \\
12.04\end{array}$ & $136.957^{\bullet}$ & \begin{tabular}{|c|}
0.00 \\
1
\end{tabular} & $\begin{array}{c}0.00 \\
5\end{array}$ & $\begin{array}{c}0.00 \\
1\end{array}$ & 0.001 \\
\hline $\begin{array}{c}\text { Creatinine } \\
(\mathrm{mg} / \mathrm{dl})\end{array}$ & $\begin{array}{c}\text { Mean } \pm S \\
\text { D }\end{array}$ & $6.54 \pm 1.75$ & $6.83 \pm 1.78$ & $0.80 \pm 0.21$ & $111.038 \bullet$ & $\begin{array}{c}0.00 \\
1\end{array}$ & \begin{tabular}{|c|}
0.46 \\
8
\end{tabular} & $\begin{array}{c}0.00 \\
1\end{array}$ & 0.001 \\
\hline $\mathrm{HB}(\mathrm{g} / \mathrm{dl})$ & $\begin{array}{c}\text { Mean } \pm S \\
\text { D } \\
\end{array}$ & $10.12 \pm 1.72$ & $10.00 \pm 1.39$ & $12.74 \pm 0.69$ & $27.539 \bullet$ & $\begin{array}{c}0.00 \\
1 \\
\end{array}$ & \begin{tabular}{|c|}
0.74 \\
8 \\
\end{tabular} & $\begin{array}{c}0.00 \\
1 \\
\end{array}$ & 0.001 \\
\hline $\mathrm{Na}^{+}(\mathrm{mEq} / \mathrm{l})$ & $\begin{array}{c}\text { Mean } \pm S \\
\text { D }\end{array}$ & $\begin{array}{c}141.13 \pm \\
3.32\end{array}$ & $\begin{array}{c}141.10 \pm \\
3.06\end{array}$ & $\begin{array}{c}139.90 \pm \\
2.90\end{array}$ & $1.141 \bullet$ & $\begin{array}{c}0.32 \\
5\end{array}$ & $\begin{array}{c}0.69 \\
7\end{array}$ & $\begin{array}{c}0.17 \\
5\end{array}$ & 0.187 \\
\hline $\mathrm{Ca}^{+}(\mathrm{mg} / \mathrm{dl})$ & $\begin{array}{c}\text { Mean } \pm S \\
\text { D }\end{array}$ & $8.63 \pm 1.00$ & $8.55 \pm 1.00$ & $9.39 \pm 0.56$ & $5.837 \bullet$ & \begin{tabular}{|c|}
0.00 \\
4
\end{tabular} & $\begin{array}{c}0.75 \\
6\end{array}$ & $\begin{array}{c}0.00 \\
5\end{array}$ & 0.002 \\
\hline
\end{tabular}

$1 \mathrm{~b}$

\begin{tabular}{|c|c|c|c|c|c|c|c|c|c|}
\hline & \multirow{2}{*}{$\begin{array}{c}\text { Obese HD } \\
\text { No. }=\mathbf{3 0} \\
\end{array}$} & \multirow{2}{*}{$\begin{array}{c}\begin{array}{c}\text { Non Obese } \\
\text { HD }\end{array} \\
\text { No. }=\mathbf{3 0} \\
\end{array}$} & \multirow{2}{*}{$\begin{array}{c}\begin{array}{c}\text { Normal } \\
\text { Obese }\end{array} \\
\text { No. }=\mathbf{2 0} \\
\end{array}$} & \multirow{2}{*}{$\begin{array}{l}\text { Test } \\
\text { value }\end{array}$} & \multirow{2}{*}{\begin{tabular}{|c|} 
P- \\
valu \\
e \\
\end{tabular}} & \multicolumn{3}{|c|}{\begin{tabular}{|c|}
$\begin{array}{c}\text { Post Hoc analysis } \\
\text { by LSD }\end{array}$ \\
\end{tabular}} \\
\hline & & & & & & & \begin{tabular}{|l|} 
P1 \\
\end{tabular} & $\mathbf{P 2}$ & P3 \\
\hline $\begin{array}{c}\text { PLT } \\
\text { (thousand } / \mathrm{cm} \\
\mathrm{m} \text { ) }\end{array}$ & $\begin{array}{c}\text { Mean } \pm S \\
\text { D }\end{array}$ & $\begin{array}{l}235.00 \pm \\
57.96\end{array}$ & $\begin{array}{c}216.07 \pm \\
77.90\end{array}$ & $\begin{array}{c}252.25 \pm \\
78.07\end{array}$ & $1.591 \bullet$ & $\begin{array}{c}0.21 \\
0\end{array}$ & $\begin{array}{c}0.30 \\
6\end{array}$ & $\begin{array}{c}0.40 \\
3\end{array}$ & 0.082 \\
\hline \multirow{2}{*}{ CRP } & & $14(46.7 \%)$ & $19(63.3 \%)$ & $12(60.0 \%)$ & \multirow{2}{*}{$1.846^{*}$} & 0.39 & 0.19 & 0.35 & \multirow{2}{*}{0.811} \\
\hline & Positive & $16(53.3 \%)$ & $11(36.7 \%)$ & $8(40.0 \%)$ & & 7 & 4 & 5 & \\
\hline $\mathrm{Po}^{+}(\mathrm{mg} / \mathrm{dl})$ & $\begin{array}{c}\text { Mean } \pm S \\
\mathrm{D}\end{array}$ & $6.32 \pm 1.60$ & $5.99 \pm 1.87$ & $4.03 \pm 0.42$ & $15.011 \bullet$ & $\begin{array}{c}0.00 \\
1 \\
\end{array}$ & \begin{tabular}{|c|}
0.40 \\
4 \\
\end{tabular} & $\begin{array}{c}0.00 \\
1 \\
\end{array}$ & 0.001 \\
\hline $\mathrm{K}^{+}(\mathrm{mEq} / \mathrm{l})$ & $\begin{array}{c}\text { Mean } \pm S \\
\text { D }\end{array}$ & $4.24 \pm 0.34$ & $4.22 \pm 0.37$ & $4.17 \pm 0.43$ & $0.232 \bullet$ & \begin{tabular}{|c|}
0.79 \\
4
\end{tabular} & $\begin{array}{c}0.81 \\
0\end{array}$ & $\begin{array}{c}0.50 \\
0\end{array}$ & 0.646 \\
\hline $\begin{array}{l}\text { Albumin } \\
\text { (g/dl) }\end{array}$ & $\begin{array}{c}\text { Mean } \pm S \\
\mathrm{D}\end{array}$ & 3.81 & $3.77 \pm 0.47$ & $4.43 \pm 0.39$ & $14.688^{\bullet}$ & \begin{tabular}{|c|}
0.00 \\
1 \\
\end{tabular} & $\begin{array}{c}0.71 \\
4 \\
\end{array}$ & $\begin{array}{c}0.00 \\
1 \\
\end{array}$ & 0.001 \\
\hline $\mathrm{WC}(\mathrm{cm})$ & $\begin{array}{c}\text { Mean } \pm S \\
\text { D }\end{array}$ & $\begin{array}{c}117.00 \pm \\
13.18\end{array}$ & $\begin{array}{c}84.73 \pm \\
11.29\end{array}$ & $\begin{array}{c}111.10 \pm \\
16.21\end{array}$ & $48.190 \bullet$ & $\begin{array}{c}0.00 \\
1\end{array}$ & $\begin{array}{c}0.00 \\
1\end{array}$ & $\begin{array}{c}0.13 \\
0\end{array}$ & 0.001 \\
\hline PTH(pg/ml) & $\begin{array}{c}\text { Median(I } \\
\text { QR })\end{array}$ & $\begin{array}{c}534.5(231- \\
1500)\end{array}$ & $\begin{array}{l}751.5(354- \\
1200)\end{array}$ & $\begin{array}{l}39.5(27- \\
53.5)\end{array}$ & $44.611 \%$ & $\begin{array}{c}0.00 \\
1\end{array}$ & $\begin{array}{c}0.60 \\
0\end{array}$ & $\begin{array}{c}0.00 \\
1\end{array}$ & 0.001 \\
\hline $\operatorname{HCT}(\%)$ & $\begin{array}{c}\text { Mean } \pm S \\
\text { D }\end{array}$ & $30.97 \pm 5.60$ & $30.61 \pm 4.80$ & $37.40 \pm 2.06$ & $15.265 \bullet$ & $\begin{array}{c}0.00 \\
1\end{array}$ & $\begin{array}{c}0.76 \\
5\end{array}$ & $\begin{array}{c}0.00 \\
1\end{array}$ & 0.001 \\
\hline PCT(pg/ml) & $\begin{array}{c}\text { Median(I } \\
\text { QR) }\end{array}$ & 178 & 106.50 & 54 & $10.120 \%$ & $\begin{array}{c}0.00 \\
1 \\
\end{array}$ & $\begin{array}{c}0.37 \\
1 \\
\end{array}$ & $\begin{array}{c}0.00 \\
1\end{array}$ & 0.031 \\
\hline
\end{tabular}

1c

Table (1): Correlation between clinical and laboratory parameters in all studied groups.

*:Chi-square test; $\bullet$ : One Way ANOVA test, $\$$ : Kruskal Wallis test, P-value $>0.05$ Non significant, $\mathrm{P}$-value $<0.05$ Significant

P-value <0.01 highly significant, P1: Obese HD vs. Non Obese HD, P2: Obese HD vs. Normal obese, P3: Non Obese HD vs. Normal obese.

We found no statistical significant difference ( $\mathrm{P}$ value $>0.05)$ between group (1) and group (2) as regard cause of renal failure and duration of HD (Table 2).

\section{\begin{tabular}{|l|l|l|l|}
\hline Obese HD & Non Obese HD & Test value & P-value \\
\hline
\end{tabular}}


Assessment of Procalcitonin Level in Chronic Hemodialysis Patients with Obesity

\begin{tabular}{|c|c|c|c|c|c|}
\hline & & No. $=30$ & No. $=30$ & & \\
\hline Cause of RF & \begin{tabular}{|c|} 
Unknown \\
Hypertension \\
DM \\
Both \\
Renal stones
\end{tabular} & $\begin{array}{c}0(0.0 \%) \\
25(83.3 \%) \\
0(0.0 \%) \\
5(16.7 \%) \\
0(0.0 \%)\end{array}$ & $\begin{array}{c}2(6.7 \%) \\
24(80.0 \%) \\
2(6.7 \%) \\
1(3.3 \%) \\
1(3.3 \%)\end{array}$ & $7.687 *$ & 0.104 \\
\hline Duration of HD(years) & $\operatorname{Median}(\mathrm{IQR})$ & $4.25(2.5-6)$ & $4.50(3-12)$ & $-0.734 t$ & 0.463 \\
\hline No. of sessions/week & 3 & $30(100.0 \%)$ & $30(100.0 \%)$ & NA & NA \\
\hline $\begin{array}{c}\text { Duration of } \\
\text { session(hours) }\end{array}$ & 4 & $30(100.0 \%)$ & $30(100.0 \%)$ & NA & NA \\
\hline
\end{tabular}

Table (2): Correlation between some clinical parameters in group (1) and group (2).

*:Chi-square test; $\$$ : Mann Whitney test, P-value $>0.05$ Non significant, P-value $<0.05$ Significant $\mathrm{P}$-value $<0.01$ highly significant.

We also found a statistical significant difference $(\mathrm{P}$ value $<0.05)$ between procalcitonin $(\mathrm{PCT})$ and age in addition to blood urea in group (1), between PCT and height in addition to HB in group (3)(Table 3).

\begin{tabular}{|c|c|c|c|c|c|c|}
\hline \multirow{2}{*}{ PCT(pg/ml) } & \multicolumn{2}{|c|}{ Obese HD } & \multicolumn{2}{c|}{ Non Obese HD } & \multicolumn{2}{c|}{ Normal Obese } \\
\cline { 2 - 7 } & r & P-value & r & P-value & r & P-value \\
\hline Age(years) & $\mathbf{0 . 4 2 2}^{*}$ & $\mathbf{0 . 0 2 0}$ & -0.328 & 0.077 & -0.359 & 0.120 \\
\hline Weight(kg) & 0.031 & 0.872 & 0.068 & 0.721 & 0.193 & 0.416 \\
\hline Height(cm) & 0.028 & 0.883 & -0.168 & 0.376 & $\mathbf{0 . 4 6 6}$ & $\mathbf{0 . 0 3 8}$ \\
\hline Duration of HD(years) & -0.042 & 0.824 & -0.321 & 0.084 & - & - \\
\hline BMI(Kg/m $\left.{ }^{2}\right)$ & -0.049 & 0.795 & 0.097 & 0.610 & -0.121 & 0.611 \\
\hline WBCS(thousand/cmm) & 0.154 & 0.416 & -0.068 & 0.723 & -0.312 & 0.180 \\
\hline RBCS(millions/cmm) & 0.083 & 0.663 & 0.244 & 0.193 & 0.048 & 0.841 \\
\hline SBP(mmHg) & 0.167 & 0.378 & -0.032 & 0.866 & -0.163 & 0.492 \\
\hline DBP(mmHg) & 0.001 & 0.994 & 0.084 & 0.659 & 0.228 & 0.334 \\
\hline Urea(mg/dl) & $\mathbf{0 . 4 1 1}^{*}$ & $\mathbf{0 . 0 2 4}$ & 0.014 & 0.943 & 0.287 & 0.220 \\
\hline Creatinine(mg/dl) & -0.072 & 0.705 & -0.050 & 0.793 & 0.325 & 0.162 \\
\hline
\end{tabular}

\section{$3 \mathrm{a}$}

\begin{tabular}{|c|c|c|c|c|c|c|}
\hline \multirow{2}{*}{ PCT $(\mathbf{p g} / \mathbf{m l})$} & \multicolumn{2}{|c|}{ Obese HD } & \multicolumn{2}{c|}{ Non Obese HD } & \multicolumn{2}{c|}{ Normal Obese } \\
\cline { 2 - 7 } & $\mathbf{r}$ & $\mathbf{P}$-value & $\mathbf{r}$ & P-value & r & P-value \\
\hline $\mathrm{HB}(\mathrm{g} / \mathrm{dl})$ & 0.186 & 0.326 & 0.159 & 0.400 & $\mathbf{0 . 6 8 2}^{* *}$ & $\mathbf{0 . 0 0 1}$ \\
\hline $\mathrm{Na}^{+}(\mathrm{mEq} / \mathrm{l})$ & -0.077 & 0.687 & 0.194 & 0.304 & 0.300 & 0.198 \\
\hline $\mathrm{Ca}^{+}(\mathrm{mg} / \mathrm{dl})$ & 0.102 & 0.593 & -0.160 & 0.399 & -0.412 & 0.071 \\
\hline PLT(thousand/cmm) & 0.009 & 0.964 & -0.004 & 0.981 & 0.038 & 0.872 \\
\hline Po4 ${ }^{+}(\mathrm{mg} / \mathrm{dl})$ & -0.325 & 0.079 & 0.037 & 0.848 & 0.296 & 0.205 \\
\hline $\mathrm{K}^{+}(\mathrm{mEq} / \mathrm{l})$ & -0.047 & 0.807 & 0.063 & 0.741 & -0.285 & 0.223 \\
\hline $\left.\mathrm{Albumin}_{\mathrm{yl}} / \mathrm{dl}\right)$ & -0.147 & 0.440 & -0.124 & 0.515 & 0.266 & 0.257 \\
\hline $\mathrm{PTH}(\mathrm{pg} / \mathrm{ml})$ & -0.232 & 0.217 & -0.074 & 0.696 & 0.369 & 0.110 \\
\hline $\mathrm{HCT}(\%)$ & 0.227 & 0.227 & 0.150 & 0.428 & $\mathbf{0 . 5 9 3}$ & $\mathbf{0 . 0 0 6}$ \\
\hline $\mathrm{WC}(\mathrm{cm})$ & 0.041 & 0.831 & -0.099 & 0.604 & -0.256 & 0.276 \\
\hline
\end{tabular}

Table (3): Correlation between procalcitonin and all the parameters in all studied groups. 


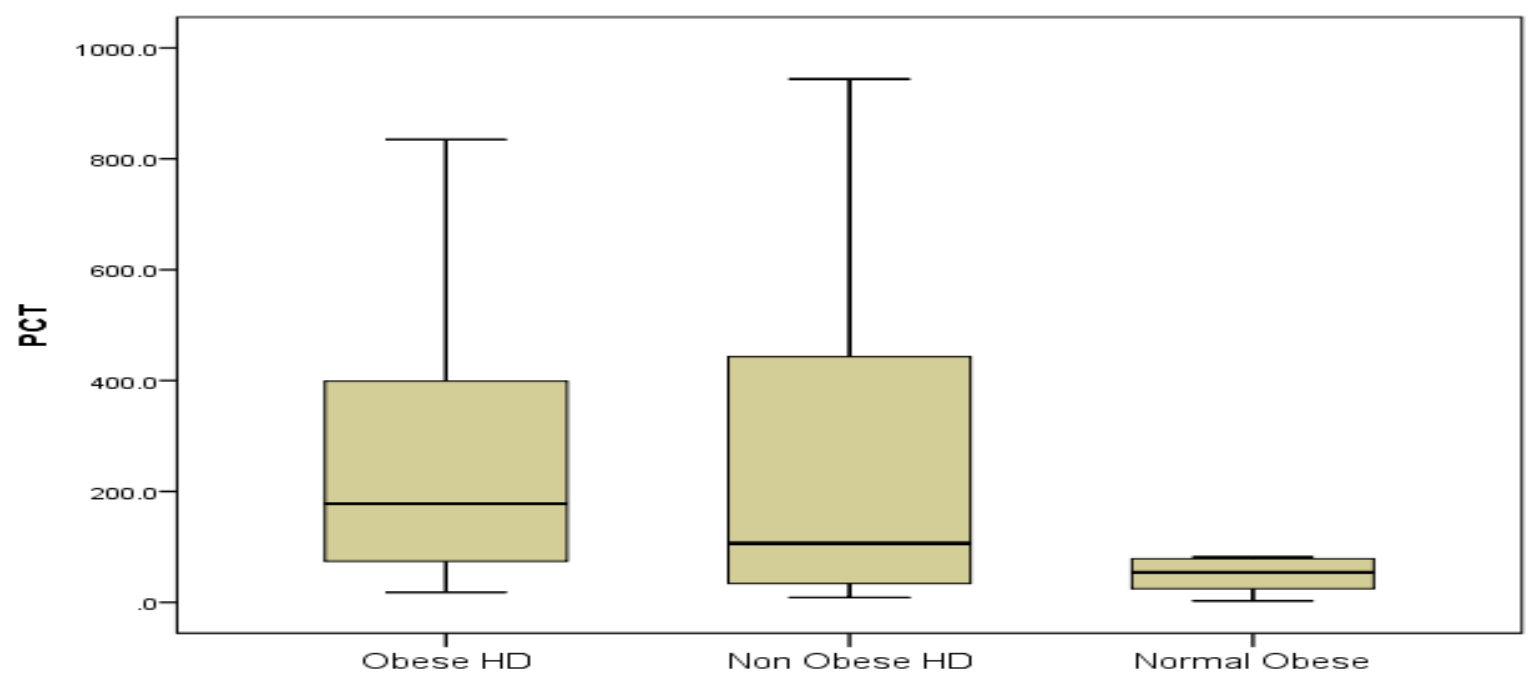

Figure (1): comparison between 3 groups as regard the median of procalcitonin hormone (PCT)

In our study, we found a highly statistical significant difference $(\mathrm{P}$ value $<0.01)$ between 3 groups regarding the mean of procalcitonin(PCT) as it increased within the normal range (Figure 1).

\section{Discussion}

Procalcitonin (PCT) high levels are strongly associated with systemic bacterial infections ${ }^{(1)}$ and with the severity of illness ${ }^{(3)}$. It is produced in response to bacterial endotoxin and inflammatory host cytokines and may help in discriminating bacterial from viral infections (4) and true bacteremia from contaminated blood cultures ${ }^{(11,12)}$.

A significant number of patients who undergo hemodialysis are overweight or obese ${ }^{(9)}$. The presence of excess weight, especially visceral obesity contributes to the increased risk of metabolic and cardiovascular complications in patients with chronic kidney disease (CKD). Some studies showed that adipose tissue is capable of expressing and secreting procalcitonin (11). So we studied the association of plasma procalcitonin with measures of obesity in chronic renal failure patients undergoing haemodialysis.

We found that procalcitonin was not dependent on the body mass index in any of the three groups as there was no any statistical difference between them ${ }^{(13)}$. This is in contrary to Abbasi et al. ${ }^{(14)}$ who found that PCT increased gradually within the normal range according to increase in body mass index.

Another result in this study was that PCT was not dependent on waist circumference in any group ( $\mathrm{P}$ value $>0.05$ ). On the other hand Abbasi et al. (14) showed that PCT increased gradually within the normal range according to increase in mean of waist circumference.

Our study showed that procalcitonin had a statically significant positive correlation with age in group (1) ( $\mathrm{P}$ value $<0.05$ ) which comes in agree with ${ }^{(14)}$.There was also no significant correlation between PCT and weight in all groups ${ }^{(15)}$.

Also, our results revealed that there was no significant correlation between PCT and duration of HD in group (1) and group (2) ${ }^{(\mathbf{1 6})}$. Concerning systolic and diastolic blood pressure, our study showed no significant correlation between them and PCT which comes in agree with ${ }^{(\mathbf{1 3}, 16)}$. In addition we found that the increase in PCT was significantly correlated ( $\mathrm{P}$ value $<0.05)$ to the increase in blood urea in group (1) ${ }^{(17)}$.

Serum sodium and potassium in this study had no correlation with PCT in all studied groups ${ }^{(15)}$. The dialysis coefficient and proper management of the patients concerning medication and life style may play a role in this.

No significant correlation was found between PCT and WBCs count, RBCs count and heamoglobin ${ }^{(\mathbf{1 8})}$. 
Al-Hakeim et al..$^{(19)}$, Kim et al. ${ }^{(15)}$ found no correlation between PCT and serum albumin which our study came in contact with it as there was no statistical difference between the two parameters in all studied groups ${ }^{(15,19)}$.

Concerning height and serum calcium, our study found no relation between PCT and the two items (19).

\section{Conclusion}

Our findings based on community-based data showed that higher plasma procalcitonin levels in the normal range are associated with increased measures of obesity. Because associations were not dependent on BMI, plasma procalcitonin may serve as a new marker for adipocyte dysfunction, chronic low-grade inflammation, or both.

1.Becker KL, Snider R and Nylen E (2010): Procalcitonin in sepsis and systemic inflammation: a harmful biomarker and a therapeutic target. British Journal of Pharmacology, 159(2): 253-264.

2.Russell J (2006): Management of sepsis. New England Journal of Medicine, 355(16): 1699-1713.

3.Angus DC and Van der Poll T (2013): Severe sepsis and septic shock. New England Journal of Medicine, 369(9): 840-851.

4. Simon L, Gauvin F, Amre DK et al. (2004): Serum procalcitonin and C-reactive protein levels as markers of bacterial infection: a systematic review and metaanalysis. Clinical Infectious Diseases, 39(2): 206-217.

5. Shomali W, Hachem R, Chaftari AM et al. (2013): Can procalcitonin differentiate Staphylococcus aureus from coagulasenegative staphylococci in clustered grampositive bacteremia? Diagnostic Microbiology and Infectious Disease, 76(2): 158-161.

6. Kumar S, Ingle H, Prasad DV et al. (2013): Recognition of bacterial infection by innate immune sensors. Critical Reviews in Microbiology, 39(3): 229-246.

7. Dandona $\mathrm{P}$, Nix D, Wilson MF et al. (1994): Procalcitonin increase after endotoxin injection in normal subjects. The
Journal of Clinical Endocrinology \& Metabolism, 79(6): 1605-1608.

8. Meisner M (2010): Procalcitoninbiochemistry and clinical diagnosis. website: https://www.unimed.de/procalcitonin-biochemistry-andclinical-diagnosis-5034.html

9. Yang SY, Chiang CK, Hsu SP et al. (2007): Metabolic syndrome predicts hospitalization in hemodialysis patients: a prospective Asian cohort study. Blood Purification, 25(3): 252-259.

10. Panzetta $G$ and Abaterusso $C$ (2010): Obesity in dialysis and reverse epidemiology: True or false? G. Ital. Nefrol., 27(6):629-38.

11. Linscheid P, Seboek D, Zulewski H et al. (2005): Autocrine/paracrine role of inflammation-mediated calcitonin generelated peptide and adrenomedullin expression in human adipose tissue. Endocrinology, 146(6): 2699-2708.

12. Schuetz P, Mueller B and Trampuz A (2007): Serum procalcitonin for discrimination of blood contamination from bloodstream infection due to coagulasenegative staphylococci. Infection, 35(5): 352-355.

13. Baghi HM (2015): Evaluation Serum Procalcitonin Level in Febrile Patients in Emergency Ward. Int. J. Curr. Microbiol. App. Sci., 4(11): 875-880.

14. Abbasi A, Corpeleijn E, Postmus D et al. (2010): Plasma procalcitonin is associated with obesity, insulin resistance, and the metabolic syndrome. The Journal of Clinical Endocrinology \& Metabolism, 95(9): 26-31.

15. Kim Y, Ji-Yoon S, Kyung-Yae $\mathrm{H}$ et al. (2016): Correlation of Glasgow Prognostic Score or Procalcitonin to Clinical Variables in Patients with Pretreatment Lung Cancer. Biomedical Science Letters, 22(1): 9-17.

16. Ichihara K, Toshiaki T, Satoshi T et al. (2016): Serum procalcitonin level in chronic hemodialytic patients with no evidence of bacterial infection. Renal Replacement Therapy, 2(1): 9-18.

17. Amour J, Birenbaum A, Langeron $\mathrm{O}$ et al. (2008): Influence of renal dysfunction on the accuracy of procalcitonin for the diagnosis of postoperative infection after vascular 
surgery. Critical Care Medicine, 36(4): 1147-1154.

18. Abedini M, Delpisheh A, Nikkhu B et al. (2012): Procalcitonin and white blood cell count (WBC), erythrocyte sedimentation rate (ESR) and serum C-reactive protein (CRP) alterations in children with systemic inflammatory response syndrome before and after treatment. African Journal of Biotechnology, 11(49): 10989-10993.

19. Al-Hakeim HK, Al-Hillawi ZM and AlKindi MA (2015): Procalcitonin, Calcium, and Magnesium in Patients with Febrile Seizure during One-Hour Attack. British Journal of Medicine and Medical Research, 6(6): 10989-10993. 